\title{
The Drift Chamber detector of the FOOT experiment: Performance analysis and external calibration
}

Dong Yunsheng ${ }^{8,23, *}$, Silvestre Gianluigi ${ }^{11,24}$, Colombi Sofia ${ }^{15,16}$, Alexandrov Andrey ${ }^{10,19,33,34}$, Alpat Behcet ${ }^{11}$, Ambrosi Giovanni ${ }^{11}$, Argirò Stefano ${ }^{28,17}$, Arteche Diaz Raul ${ }^{30}$, Barbanera Mattia ${ }^{2,35}$, Bartosik Nazar ${ }^{17}$, Belcari Nicola ${ }^{3,2}$, Bellinzona Elettra ${ }^{15}$, Biondi Silvia ${ }^{5,20}$, Bisogni Maria Giuseppina ${ }^{3,2}$, Bruni Graziano ${ }^{5}$, Carra Pietro ${ }^{3,2}$, Cerello Piergiorgio ${ }^{17}$, Ciarrocchi Esther ${ }^{3,2}$, Clozza Alberto ${ }^{7}$, De Lellis Giovanni ${ }^{10,19}$, Del Guerra Alberto ${ }^{3,2}$, De Simoni Micol ${ }^{12,26}$, Di Crescenzo Antonia ${ }^{10,19}$, Di Ruzza Benedetto ${ }^{15}$, Donetti Marco ${ }^{17,1}$, Durante Marco ${ }^{6,32}$, Ferrero Veronica ${ }^{17}$, Fiandrini Emanuele ${ }^{11,24}$, Finck Christian ${ }^{14}$, Fiorina Elisa ${ }^{17}$, Fischetti Marta ${ }^{12,22}$, Francesconi Marco ${ }^{3,2}$, Franchini Matteo ${ }^{5,20}$, Franciosini Gaia ${ }^{12,26}$, Galati Giuliana ${ }^{10}$, Galli Luca ${ }^{2}$, Gentile Valerio ${ }^{31}$, Giraudo Giuseppe ${ }^{17}$, Hetzel Ronja ${ }^{4}$, Iarocci Enzo ${ }^{7}$, Ionica Maria ${ }^{11}$, Kanxheri Keida ${ }^{11}$, Kraan Aafke Christine ${ }^{2}$, Lante Valeria $^{1}$, La Tessa Chiara ${ }^{15,16}$, Laurenza Martina ${ }^{7}$, Lauria Adele ${ }^{10,19}$, Lopez Torres Ernesto $^{30,17}$, Marafini Michela ${ }^{12,21}$, Massimi Cristian ${ }^{20}$, Mattei Ilaria ${ }^{8}$, Mengarelli Alberto $^{5}$, Moggi Andrea ${ }^{2}$, Montesi Maria Cristina ${ }^{10,19}$, Morone Maria Cristina ${ }^{13,27}$, Morrocchi Matteo $^{2,3}$, Muraro Silvia ${ }^{8}$, Narici Livio ${ }^{13,27}$, Pastore Alessandra ${ }^{29}$, Pastrone Nadia ${ }^{17}$, Patera Vincenzo ${ }^{12,22}$, Pennazio Francesco ${ }^{17}$, Placidi Pisana ${ }^{11,25}$, Pullia Marco ${ }^{1}$, Raffaelli Fabrizio $^{2}$, Ramello Luciano ${ }^{18,17}$, Ridolfi Riccardo ${ }^{20,5}$, Rosso Valeria ${ }^{3,2}$, Sanelli Claudio $^{7}$, Sarti Alessio ${ }^{12,22}$, Sartorelli Gabriella ${ }^{5,20}$, Sato Osamu ${ }^{9}$, Savazzi Simone ${ }^{1}$, Scavarda Lorenzo ${ }^{28,17}$, Schiavi Angelo ${ }^{12,22}$, Schuy Christoph ${ }^{6}$, Scifoni Emanuele ${ }^{15}$, Sciubba Adalberto ${ }^{7,22}$, Sécher Alexandre ${ }^{14}$, Selvi Marco ${ }^{5}$, Sitta Mario ${ }^{18,17}$, Spighi Roberto ${ }^{5}$, Spiriti Eleuterio ${ }^{7}$, Sportelli Giancarlo ${ }^{3,2}$, Stahl Achim ${ }^{4}$, Tomassini Sandro ${ }^{7}$, Toppi Marco ${ }^{7,22}$, Traini Giacomo ${ }^{12,26}$, Valeri Tioukov ${ }^{10}$, Valle Serena Marta ${ }^{8}$, Marie Vanstalle ${ }^{14}$, Mauro Villa ${ }^{5,20}$, Weber Ulrich $^{6}$, Zoccoli Antonio ${ }^{5,20}$, Battistoni Giuseppe ${ }^{8}$, Servoli Leonello ${ }^{11}$, Tommasino Francesco ${ }^{15,16}$

\footnotetext{
${ }^{1}$ Centro Nazionale di Adroterapia Oncologica (CNAO), Pavia, Italy

${ }^{2}$ Istituto Nazionale di Fisica Nucleare (INFN), Section of Pisa, Pisa, Italy

${ }^{3}$ University of Pisa, Department of Physics, Pisa, Italy

${ }^{4}$ RWTH Aachen University, Physics Institute III B, Aachen, Germany

${ }^{5}$ Istituto Nazionale di Fisica Nucleare (INFN), Section of Bologna, Bologna, Italy

${ }^{6}$ Biophysics Department, GSI Helmholtzzentrum für Schwerionenforschung, Darmstadt, Germany

${ }^{7}$ Istituto Nazionale di Fisica Nucleare (INFN), Laboratori Nazionali di Frascati, Frascati, Italy

${ }^{8}$ Istituto Nazionale di Fisica Nucleare (INFN), Section of Milano, Milano, Italy

${ }^{9}$ Nagoya University, Department of Physics, Nagoya, Japan

${ }^{10}$ Istituto Nazionale di Fisica Nucleare (INFN), Section of Napoli, Napoli, Italy

${ }^{11}$ Istituto Nazionale di Fisica Nucleare (INFN), Section of Perugia, Perugia, Italy

${ }^{12}$ Istituto Nazionale di Fisica Nucleare (INFN), Section of Roma 1, Rome, Italy

${ }^{13}$ University of Rome Tor Vergata, Department of Physics, Rome, Italy

${ }^{14}$ Université de Strasbourg, CNRS, IPHC UMR 7871, F-67000 Strasbourg, France

${ }^{15}$ Trento Institute for Fundamental Physics and Applications, Istituto Nazionale di Fisica Nucleare (TIFPA-INFN), Trento, Italy

${ }^{16}$ University of Trento, Department of Physics, Trento, Italy

${ }^{17}$ Istituto Nazionale di Fisica Nucleare (INFN), Section of Torino, Torino, Italy

${ }^{18}$ University of Piemonte Orientale, Department of Science and Technological Innovation, Alessandria, Italy
}

\footnotetext{
* Corresponding author at: University of Milano, Department of Physics, Milano, Italy. E-mail address: yunsheng.dong@mi.infn.it (Dong Y.).
} 
${ }^{19}$ University of Napoli, Department of Physics "E. Pancini", Napoli, Italy

${ }^{20}$ University of Bologna, Department of Physics and Astronomy, Bologna, Italy

${ }^{21}$ Museo Storico della Fisica e Centro Studi e Ricerche Enrico Fermi, Rome, Italy

${ }^{22}$ University of Rome La Sapienza, Department of Scienze di Base e Applicate per l'Ingegneria (SBAI), Rome, Italy

${ }^{23}$ University of Milano, Department of Physics, Milano, Italy

${ }^{24}$ University of Perugia, Department of Physics and Geology, Perugia, Italy

${ }^{25}$ University of Perugia, Department of Engineering, Perugia, Italy

${ }^{26}$ University of Rome La Sapienza, Department of Physics, Rome, Italy

${ }^{27}$ Istituto Nazionale di Fisica Nucleare (INFN), Section of Roma Tor Vergata, Rome, Italy

${ }^{28}$ University of Torino, Department of Physics, Torino, Italy

${ }^{29}$ Istituto Nazionale di Fisica Nucleare (INFN), Section of Bari, Bari, Italy

${ }^{30}$ CEADEN, Centro de Aplicaciones Tecnologicas y Desarrollo Nuclear, Havana, Cuba

${ }^{31}$ Gran Sasso Science Institute, L'Aquila, Italy

${ }^{32}$ Technische Universität Darmstadt Institut für Festkörperphysik, Darmstadt, Germany

${ }^{33}$ National University of Science and Technology, MISIS, RUS-119049 Moscow, Russia

${ }^{34}$ Lebedev Physical Institute of the Russian Academy of Sciences, RUS-119991 Moscow, Russia

${ }^{35}$ University of Pisa, Department of Information Engineering, Pisa, Italy

\section{A R T I C L E I N F O}

\section{Keywords:}

Drift chamber

Silicon microstrip detector

Nuclear physics

\begin{abstract}
A B S T R A C T
The study that we present is part of the preparation work for the setup of the FOOT (FragmentatiOn Of Target) experiment whose main goal is the measurement of the double differential cross sections of fragments produced in nuclear interactions of particles with energies relevant for particle therapy. The present work is focused on the characterization of the gas-filled drift chamber detector composed of 36 sensitive cells, distributed over two perpendicular views. Each view consists of six consecutive and staggered layers with three cells per layer. We investigated the detector efficiency and we performed an external calibration of the space-time relations at the level of single cells. This information was then used to evaluate the drift chamber resolution. An external tracking system realized with microstrip silicon detectors was adopted to have a track measurement independent on the drift chamber. The characterization was performed with a proton beam at the energies of 228 and $80 \mathrm{MeV}$. The overall hit detection efficiency of the drift chamber has been found to be $0.929 \pm 0.008$, independent on the proton beam energy. The spatial resolution in the central part of the cell is about $150 \pm 10 \mu \mathrm{m}$ and $300 \pm 10 \mu \mathrm{m}$ and the corresponding detector angular resolution has been measured to be $1.62 \pm 0.16 \mathrm{mrad}$ and $2.1 \pm 0.4 \mathrm{mrad}$ for the higher and lower beam energies, respectively. In addition, the best value on the intrinsic drift chamber resolution has been evaluated to be in the range $60-100 \mu \mathrm{m}$. In the framework of the FOOT experiment, the drift chamber will be adopted in the pre-target region, and will be exploited to measure the projectile direction and position, as well as for the identification of pre-target fragmentation events.
\end{abstract}

\section{Introduction}

Charged Particle Therapy (CPT) is an established therapeutic option for cancer treatment that exploits accelerated light charged ions (such as protons and carbon ions) for the irradiation of solid tumors. The main advantages of CPT with respect to the traditional X-ray therapy is the depth-dose curve typical of charged particles: the largest fraction of dose is released at the end of the particle path, in correspondence of the so-called Bragg Peak, thus allowing the concentration of the dose in the tumor volume, while sparing the surrounding healthy tissues. In addition to that, charged particles are characterized by an enhanced biological effectiveness, that can be evaluated by means of the Relative Biological Effectiveness (RBE), defined as the ratio of photon to charged particle physical dose resulting into the same biological effect. The RBE values depend on several biological and physical parameters, as well as on the particle type. In clinical practice, the RBE of protons is assumed to be 1.1, even though recent studies indicate comparably large RBE variability in several tissues [1]. Among the causes of this variability, one potential contribution originates from the dose deposited by the fragments produced by the nuclear interactions between the incident particles and the human body [2]. In CPT with protons, the fragments produced from the target nuclei have high charge, low kinetic energy and a range of the order of few micrometers [2]. Therefore, they can be associated to high RBE values and their damage is confined to a limited number of cells. Due to the experimental difficulties in the detection of such particles, there is a lack of accurate cross section data describing target fragments production, excluding the possibility to consider this effect in the current clinical treatment planning.

The FOOT (FragmentatiOn Of Target) experiment aims at measuring the double differential cross sections with respect to kinetic energy and emission angle of fragments produced in nuclear interactions of particles with energies relevant for proton therapy. In order to overcome the detection difficulties due to the few micron range of fragments, FOOT will adopt an inverse kinematic approach, studying the fragmentation of different ion beams (e.g. ${ }^{12} \mathrm{C},{ }^{16} \mathrm{O},{ }^{40} \mathrm{Ca}$ ) onto hydrogen enriched targets, such as $\mathrm{C}_{2} \mathrm{H}_{4}$, as already adopted in [3,4]. Secondary fragments will thus have boosted energy and longer range, making possible the detection. The final goal of the FOOT experiment will be the measurement of the fragments cross section with maximum uncertainty of $5 \%$ and of fragment energy spectra with an energy resolution of the order of $1-2 \mathrm{MeV} / \mathrm{u}$, as well as the charge and isotopic identification (at the level of $3 \%$ and $5 \%$ uncertainty, respectively). The choice of the inverse kinematics approach requires the measurement of the projectile direction and position before and after the target with an angular resolution of the order of few mrad and a spatial resolution of the order of few hundreds of $\mu \mathrm{m}$. For this reason, a Beam Monitor (BM) drift chamber detector has been adopted in the pre-target region, which will allow the determination of the Lorentz boost direction to be applied to the produced fragments. The FOOT BM detector is inherited from the FIRST experiment [5]. It has been employed in the past, adopting the space-time relations calculated using a self-calibration iterative algorithm based on the BM tracks and residuals. In order to improve the evaluation of the space-time relations and the detector performance assessment, a calibration of the space-time relations by means of an external independent tracking detector is mandatory. In this way, it is possible to overcome the difficulties related to the use of a selfcalibration algorithm whose results depend on the initial space-time relation hypothesis and on the BM track reconstruction performances. Moreover, the stopping time of the iterative procedure cannot be well determined, since artifacts introduced by the algorithm itself appear 


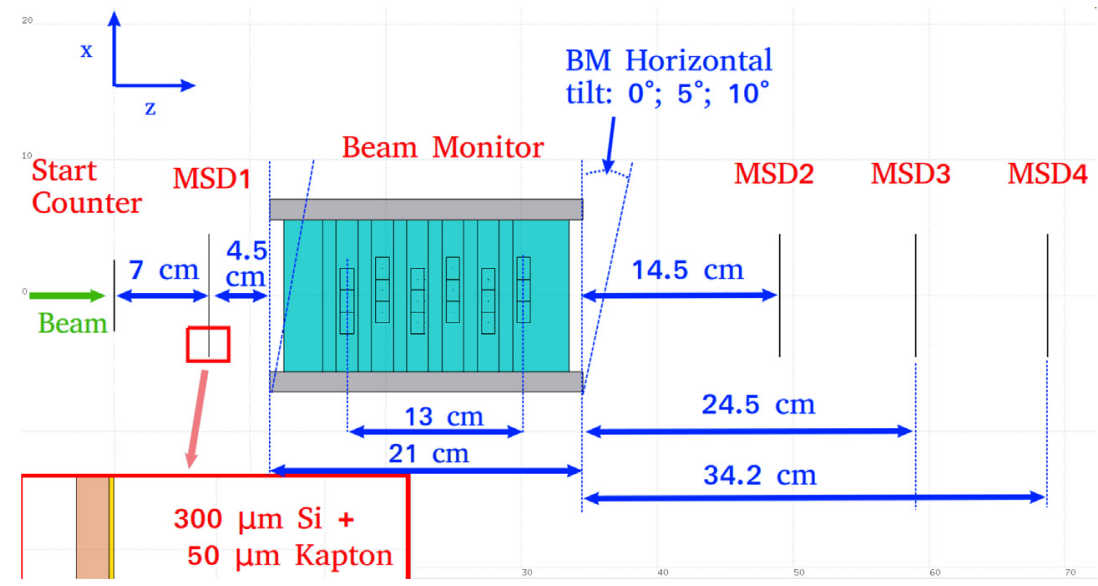

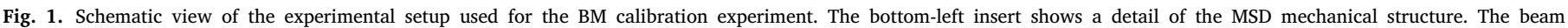
direction is horizontal from left to right.

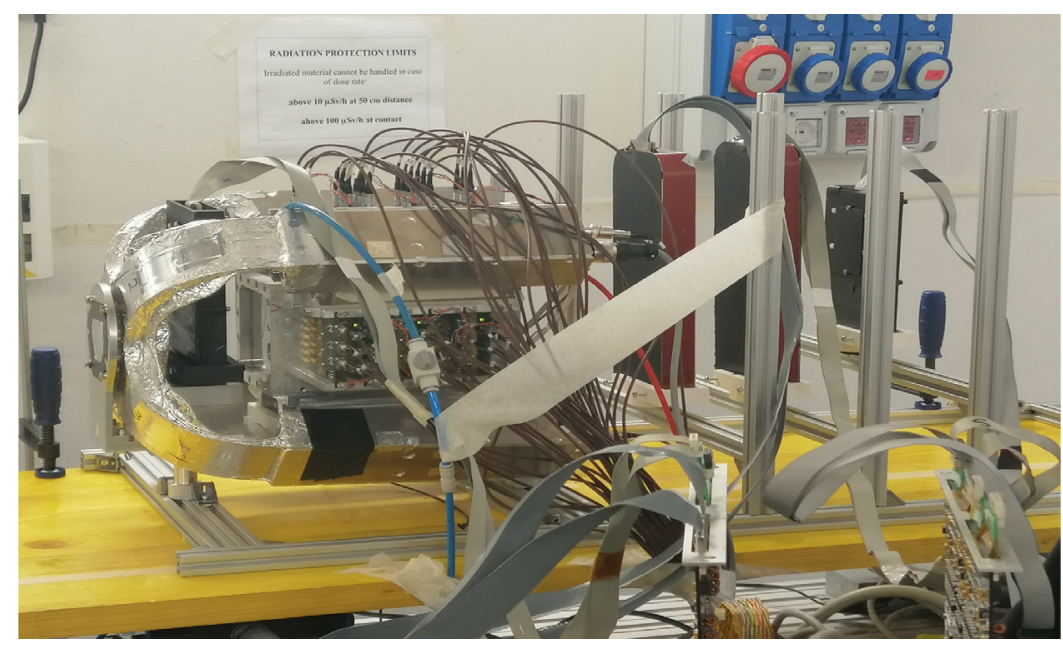

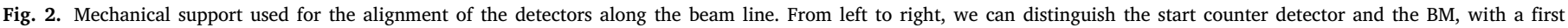
MSD (black) positioned between the two detectors (see also Fig. 1 for comparison). Downstream the BM, three MSD are aligned.

after multiple iterations. In this work we present the calibration of the space-time relations and the performance assessment of the BM of the FOOT experiment by means of Microstrip Silicon Detectors (MSD) [6], performed at the Trento proton therapy facility with protons at 80 and $228 \mathrm{MeV}$ of kinetic energy.

\section{Materials and methods}

\subsection{Experimental setup}

The BM calibration experiment was performed in the experimental area of the Trento Proton Therapy facility, where a proton beam in the energy range $80-228 \mathrm{MeV}$ is available [7]. The setup was assembled on the 30 degree beam line. It includes a plastic scintillator Start Counter (SC) detector, the BM detector and four layers of MSD. The center of the SC was placed at the isocenter of the beam, followed by one layer of MSD placed between SC and BM, and the other three placed after the BM. A schematic view of the apparatus is shown in Fig. 1, where distances between detectors are also indicated. A dedicated mechanical support was used to properly align the detectors along the beam line (Fig. 2). A detailed description of all detector types is presented below.
The start counter detector. The SC has been used in the FIRST experiment [8], and it consists of a circular $250 \mu \mathrm{m}$ thick plastic scintillator disk (EJ-228), with a $26 \mathrm{~mm}$ radius. The light produced is collected radially by 160 optical fibers grouped in four bundles and readout by fast photomultipliers (Hamamatsu H10721-201) with $40 \%$ quantum efficiency. The scintillator thickness was minimized in order to reduce the probability of particle interaction before the target. As calculated by means of MC simulations, assuming an incident beam of oxygen ions with an initial kinetic energy of $400 \mathrm{MeV} / \mathrm{u}$ delivered on a $5 \mathrm{~mm}$ thick graphite target, about $3.5 \%$ fragmentation probability is expected on the SC with respect to on-target fragmentation. A time resolution of the order of $\sigma_{t}=150 \pm 5$ ps has been measured fitting the time difference distribution between two selected photomultipliers with a Gaussian function [9]. The SC is adopted as trigger detector for the whole acquisition system and as reference time detector for the BM measurements.

The beam monitor detector. The BM is a drift chamber consisting of six planes of alternated horizontal and vertical layers of cells, closed by two mylar windows with $100 \mu \mathrm{m}$ thickness placed at the beam entrance and exit to contain the gas mixture. Each plane is composed of two layers, one with horizontal and one with vertical wires, both perpendicular 
with respect to the beam line. Each layer consists of three drift cells and two consecutive layers of the same view are staggered by half a cell in order to minimize the tracking ambiguities. The cell shape is rectangular $(16 \mathrm{~mm} \times 10 \mathrm{~mm}$ ) with the longer side orthogonal to the beam. The cell area is delimited by eight aluminum field wires with a diameter of $90 \mu \mathrm{m}$. At the cell center, a gold-plated tungsten sense wire with a diameter of $25 \mu \mathrm{m}$ is connected to the BM high voltage and it is read out by a TDC. Perpendicular to the beam direction, the overall BM active area is of $5.6 \times 5.6 \mathrm{~cm}^{2}$, but the area in which all the six planes of cells can be exploited is of the order of $4 \times 4 \mathrm{~cm}^{2}$. The outer border of the overall active area is covered by three planes of cells due to the layer staggering. Along the beam direction, the detector total length is $21 \mathrm{~cm}$ and the active length, determined as the distance between the sense wire positions on the first and the last layer, is of $13 \mathrm{~cm}$ for each view. The SC trigger signal is also acquired by the TDC in order to get a reference for the BM time measurements. During testing the $\mathrm{BM}$ has been continuously flushed with an $\mathrm{Ar} / \mathrm{CO}_{2}$ gas mixture at $80 \% / 20 \%$ and $\sim 0.9$ bar relative pressure.

The microstrip silicon detectors. The detectors employed for the calibration data taking are composed of $300 \mu \mathrm{m}$ thick double sided silicon micro strip sensors with either one (for the outermost ones, with a resulting $4 \times 7 \mathrm{~cm}^{2}$ active area) or two daisy-chained modules (for the innermost ones, with a resulting $8 \times 7 \mathrm{~cm}^{2}$ active area). The modules are directly derived from the ones used in the AMS-02 experiment [6] with the use of a new version of the readout chip (VA140, Ideas) and the reduced readout pitch on the ohmic side $(104 \mu \mathrm{m}$ vs. $208 \mu \mathrm{m})$ for the modules with a single sensor. The visible side is the p-side (junction side or S-side) while the reverse side corresponds to the n-side (ohmic side or K-side). The VA140 chip is capable or accepting either positive or negative signals, hence the only difference between the two sides is the number of readout channels and thus the number of chips. In total, each module provides 1024 readout channels, 640 for the S-side, 384 for the K-side. This results in a final spatial resolution of less than $30 \mu \mathrm{m}$ on the S-side and of either $60 \mu \mathrm{m}$ (for the detectors with a single sensor with reduced readout pitch) or $30 \mu \mathrm{m}$ (for the detectors with a single sensor) on the K-side. The overall mean point resolution along a track reconstructed by the detector is of $45 \mu \mathrm{m}$. The detectors are operated applying a positive voltage of typically $80 \mathrm{~V}$ on the $\mathrm{n}$-side guard ring, while connecting the p-side inner guard ring to ground. Due to the high leakage current of one of the sensors, the bias power supply connected to two of them had to be set at a slightly lower bias voltage of $50 \mathrm{~V}$, resulting in a lower depletion of the silicon bulk.

\subsection{Experimental plan}

The highest $(228 \mathrm{MeV})$ and lowest $(80 \mathrm{MeV})$ proton energies available at the Trento Protontherapy center were used for the experiment. The former is the best choice to characterize the detector minimizing the Multiple Coulomb Scattering (MCS) and to evaluate the detector resolution. The latter energy has been explored to check the detector sensitivity on ionization density and to evaluate the space-time relation differences, in order to mimic as much as possible the experiment conditions that will be met by the FOOT experiment, in which the BM will be exposed to more ionizing particles at different energies $\left({ }^{4} \mathrm{He}\right.$, ${ }^{12} \mathrm{C},{ }^{16} \mathrm{O}$ ). In the present experiment, as evaluated by means of PSTAR tables [10], the stopping power $(\mathrm{d} E / \mathrm{d} x)$ of 228 and $80 \mathrm{MeV}$ protons in the $\mathrm{BM}$ gas is 3.13 and $6.44 \mathrm{MeV} \mathrm{cm} / \mathrm{g}$ respectively.

The spot size of the $228 \mathrm{MeV}$ proton beam is about $3 \mathrm{~mm}$ [7], i.e. smaller than a BM single cell dimension. In order to reach all the cell area, the $\mathrm{BM}$ has been rotated at $0^{\circ}, 5^{\circ}$ and $10^{\circ}$ degrees along the $y$ axis for the calibration with the MSD. Furthermore, the tilt avoids the overlap of the cell anodes on one layer with the cell border of the next layer, which are the areas with the lowest resolution [11]. The MSD and the SC have not been moved during the entire data taking period.
Table 1

Summary of the data collected at the Proton Therapy Facility in Trento (Italy) for the BM calibration experiment.

\begin{tabular}{llllll}
\hline $\begin{array}{l}\text { Acquired } \\
\text { events }\end{array}$ & $\begin{array}{l}\text { Energy } \\
{[\mathrm{MeV}]}\end{array}$ & $\begin{array}{l}\text { Tilt } \\
{[\mathrm{deg}]}\end{array}$ & $\begin{array}{l}\text { BM } \\
\text { tracks }\end{array}$ & $\begin{array}{l}\text { MSD } \\
\text { tracks }\end{array}$ & $\begin{array}{l}\text { Combined } \\
\text { tracks }\end{array}$ \\
\hline $2 \cdot 10^{5}$ & 80 & 0 & 163360 & 120683 & 82404 \\
$1 \cdot 10^{5}$ & 228 & 0 & 82462 & 39726 & 25154 \\
$1 \cdot 10^{5}$ & 80 & 5 & 64640 & 56276 & 30128 \\
$1 \cdot 10^{5}$ & 228 & 5 & 78671 & 50654 & 33060 \\
$1 \cdot 10^{5}$ & 228 & 10 & 60707 & 35842 & 17971 \\
\hline
\end{tabular}

\subsection{Data selection and reconstruction}

A particle with a straight track crossing all the BM should give 12 hits in total, but due to delta rays, cross talks, detector inefficiency and geometry reasons, the hit distribution is spread. In order to avoid these effects as much as possible, but maintaining a sufficient number of events per dataset, we decided to select the events with a number of BM hits $\leq 14$ and $\geq 10$. In the selected events, the BM time measurements with $\Delta t \leq 320 \mathrm{~ns}$ are converted to distance measurements adopting the space-time relations calculated in the FIRST experiment [11]. Then, the hits are processed by a track reconstruction algorithm based on the Legendre polynomials [12]. The Legendre space is a bi-dimensional space in which the two coordinates are the two parameters of a bidimensional line. All the BM hits are represented in the Legendre space with the Legendre transform and the hits that are compatible with the same track results to be clustered. The cluster with the highest number of hits is considered as the projectile track and the cluster position gives an initial guess of the track parameters. Finally the hits associated with this cluster are given as input to a $\chi^{2}$ minimization algorithm to refine the track parameters estimation.

In order to reconstruct the MSD tracks, first an internal alignment of all the detectors is performed by means of an iterative minimization process on the residuals distributions: events to be used in this process are required to have a single reconstructed hit in each detector to eliminate track multiplicity ambiguities. To minimize the contribution of MCS and to introduce the smallest bias possible in the final track reconstruction the required events are taken from a random sub-sample of those at the highest energy available. The final tracks are then reconstructed using the two detectors closest to the BM (one upstream and one downstream) to mitigate effects from MCS on the track resolution.

The BM tracks with a reduced $\chi^{2}\left(\chi_{\text {red }}^{2}\right) \leq 10$ are selected and matched with the MSD tracks to extract the alignment parameters with an iterative method based on the minimization of the tracks residuals. The number of collected events, selected tracks, the proton beam energy and the tilting angle of the BM with respect to the beam line for the analyzed dataset are reported in Table 1.

Once the tracks are coupled, the two detector measurements are combined to assess the BM performances presented in Section 3. As shown in Table 1, the selection criteria strongly reduce the number of collected data in order to maximize the tracks precision and reliability, excluding as much as possible the contribution of the delta rays. Indeed, considering the $228 \mathrm{MeV}$ events reconstructed by the MSD and taken with the setup at $0^{\circ}$, the BM track reconstruction efficiency, evaluated as the fraction of events in which the BM reconstructed a track without any selection criteria, is of $0.9906 \pm 0.0005$. The cut on the number of hits reduce the efficiency to $0.828 \pm 0.002$ and the cut on the $\chi_{\text {red }}^{2}$ further reduce the efficiency to $0.633 \pm 0.002$. The drop of the number of $\mathrm{BM}$ reconstructed tracks in the tilted setups is due to the reduced angular acceptance of the detector with respect to the beam direction. Since the beam spot size at the isocenter is of $\sigma_{x}=2.74 \mathrm{~mm}$ and $\sigma_{x}=6.93$ $\mathrm{mm}$ for the 228 and $80 \mathrm{MeV}$ protons [7], in the latter case the efficiency drop is enhanced. However, by means of a Monte Carlo (MC) simulation performed with the FLUKA MC code [13-15], it has been previously verified that the total number of selected events is sufficient to achieve the analysis purpose. 

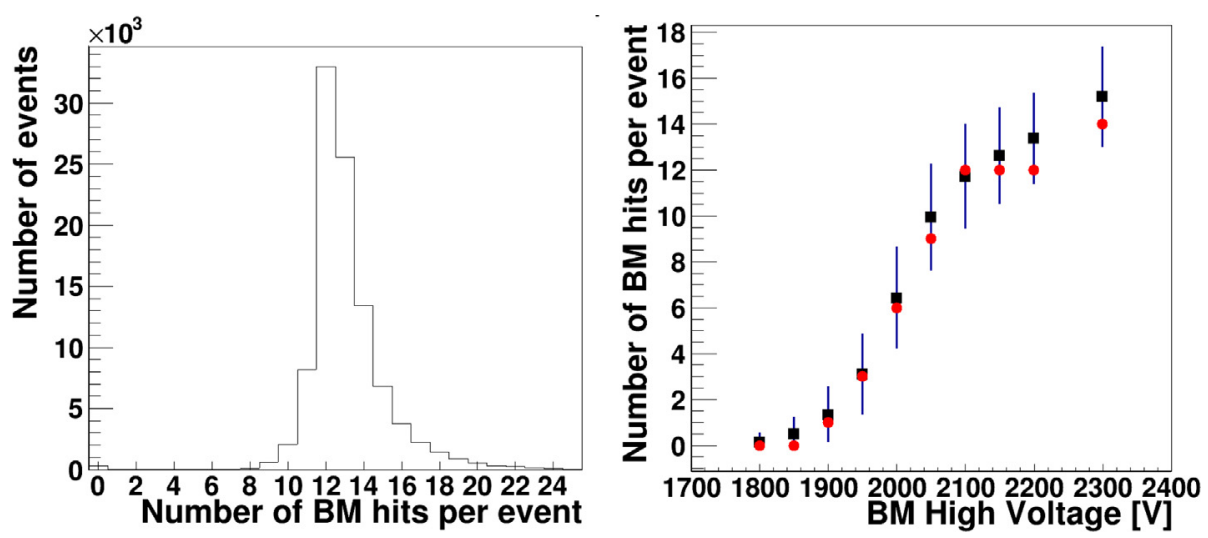

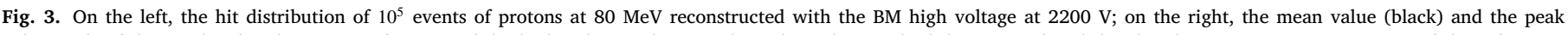

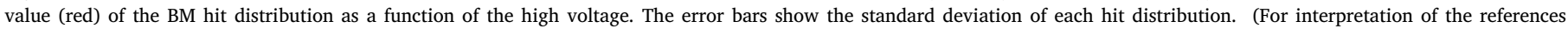
to color in this figure legend, the reader is referred to the web version of this article.)
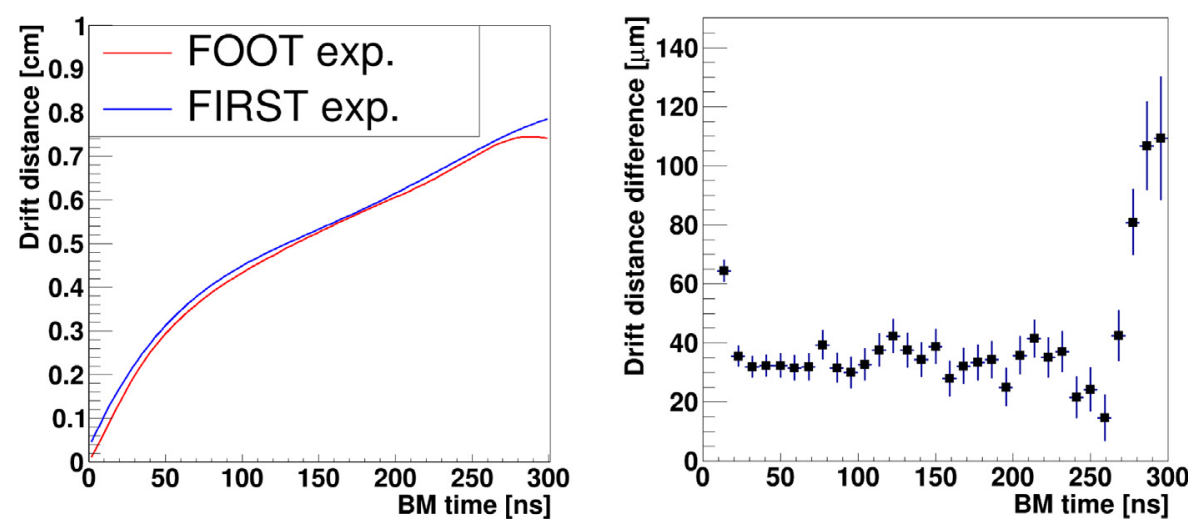

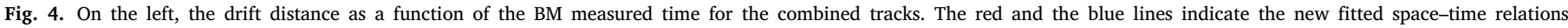

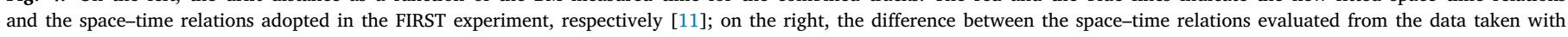

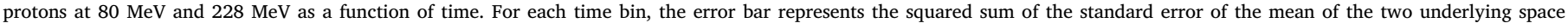
distributions. (For interpretation of the references to color in this figure legend, the reader is referred to the web version of this article.)

\section{Results}

Once the tracks are selected and the detector alignment parameters retrieved, different parameters have been studied to characterize the $\mathrm{BM}$ detector and to ensure that its performance satisfies the requirements of the FOOT experiment.

\subsection{BM hit distribution and working point}

In order to select an optimal High voltage (HV) value, the BM was first exposed to a fixed $80 \mathrm{MeV}$ proton beam and a set of data was taken varying the $\mathrm{HV}$ from 1800 to $2300 \mathrm{~V}$. A minimum of $10^{5}$ primaries was acquired for each data set. The threshold on the BM channels was set to $20 \mathrm{mV}$ during the data acquisition. This value is above the noise level and below the typical BM signal that is of the order of hundreds of $\mathrm{mV}$. An example of the hit distribution for a given HV is presented in the left panel of Fig. 3. The asymmetric shape of the distribution is given by the effect of delta rays, cross talks and geometry, since a particle can produce ionization in more than a single cell in each layer. Thus the hit distribution mean value is slightly higher than the peak value. The mean and the peak values of the number of hits as a function of the HV are shown on the right panel of Fig. 3. As expected, the number of hits increases with the increase of the HV. A minimum threshold value of $1900 \mathrm{~V}$ on the $\mathrm{HV}$ has been found to detect the $80 \mathrm{MeV}$ protons.
Since the expected number of hits for each event is 12 and in order to maintain a high level of efficiency (as shown in Section 3.3), the optimal HV working point for the BM has been found to be $2200 \mathrm{~V}$, in which the mean number of hit per event is 14 and the peak of the hit distribution is 12 hits. The same HV value has been adopted during the acquisition of protons at $228 \mathrm{MeV}$, since the same hit distribution properties have been found.

\subsection{Space-time relations}

In order to evaluate the BM space-time relations minimizing the bias due to MCS, the data taken with protons at the maximum available energy of $228 \mathrm{MeV}$ are considered. For each event that passes all the selection criteria, the MSD reconstructed track is extrapolated into the BM detector and the cells crossed by the track that contain a BM hit are considered for the analysis. For each selected cell, the distance of the track with respect to the cell sense wire position is calculated. This value represents the drift distance of the projectile and it can be coupled with the BM time measurement. The two values calculated from all the events fill a two dimensional plot that represents the distribution of the drift distance as a function of the time measurement. In order to get a clean space-time relation distribution, a profiling procedure is adopted and for each time bin, the mean value of the relative underlying space distribution is considered as the best value for the given time bin. 

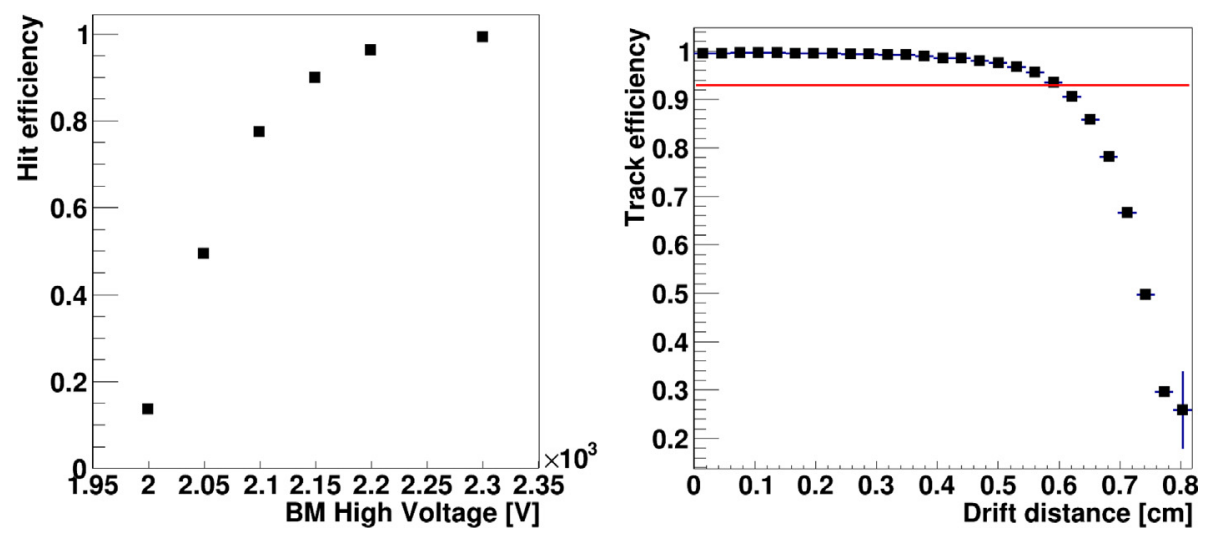

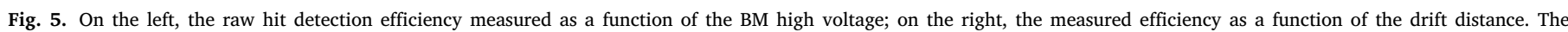

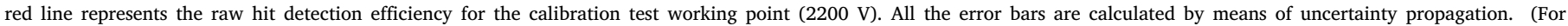
interpretation of the references to color in this figure legend, the reader is referred to the web version of this article.)

The results are shown in Fig. 4 on the left with the new fitted space-time relations in red. For comparison, the blue line represents the space-time relations adopted in the FIRST experiment [11] and evaluated with an iterative procedure based on data taken from a test with carbon ions at $80 \mathrm{MeV} / \mathrm{u}$ and the BM operating at $1800 \mathrm{~V}$. Even though the incident particles and the BM working point were different, the two curves look quite similar, since no relevant differences are observed on the reconstructed tracks and on the resolution evaluation using both space-time relations.

The space-time relation difference between the data taken with protons at 80 and $228 \mathrm{MeV}$ is shown on the right panel of Fig. 4. For a given time measurement, the corresponding drift distance is larger for the protons at the lower energy. This effect is due to the enhancement of the mean free path between two ionization clusters of the high energy-low ionizing protons with respect to the low energyhigh ionizing protons. Indeed, in the energy range considered in this analysis, the $80 \mathrm{MeV}$ protons will ionize a factor 2 more than $228 \mathrm{MeV}$ protons, providing thus more ionization clusters. In this case, the distance between the cell sense wire and the closest ionizing cluster is lower and the drift signal will be collected in less time with respect to the high energy particle. Thus, for a given time measurement, the low energy particles are associated with a larger drift distance. This effect is enhanced at the BM cell border due to the lowering of the electric field, where also time-walk and time-jitter effects on the wire signal can have a role. The difference of the space-time relations between the two proton energies data is $35 \pm 10 \mu \mathrm{m}$ for the hits with a time measurement shorter than $270 \mathrm{~ns}$ and $100 \pm 13 \mu \mathrm{m}$ for the hits associated with a time higher than 270 ns. However, in the former case it is 4-5 times smaller than the BM mean resolution at the cell center and in the latter case it is 2-3 times smaller than the resolution at the cell border. Considering also the BM inefficiency and the reduced number of hits detected at the cell border, the difference between the spacetime relations evaluated with the two proton energies is a negligible effect for the track reconstruction purpose.

\subsection{Efficiency}

In order to find a proper working point, the BM raw hit detection efficiency is estimated using a pivot and probe counter method: for each view, the pivots are the events in which three hits are detected on the three odd (or even) planes. On this subset of events, the probes are the events in which also the two even (or odd) planes placed between the pivots planes contains at least a hit. The hit detection efficiency is defined as the ratio of probes to pivots. At first, efficiency is evaluated as a function of the BM HV. For a fixed beam of protons at $80 \mathrm{MeV}$, we took a dataset of $100 \mathrm{k}$ events for each HV step of $50 \mathrm{~V}$, ranging from $1900 \mathrm{~V}$ up to the maximum voltage allowed of $2300 \mathrm{~V}$. The results are shown on the left panel of Fig. 5. As expected, the efficiency increases as the HV increases. In order to have an efficiency $\geq 0.9$, the minimum $\mathrm{HV}$ value has been found to be $2150 \mathrm{~V}$. For the HV value of $2200 \mathrm{~V}$ chosen as the optimal working point after the procedure described in Section 3.1, the efficiency is evaluated also varying the beam energy from $80 \mathrm{MeV}$ to $220 \mathrm{MeV}$ with steps of $20 \mathrm{MeV}$, taking datasets with $100 \mathrm{k}$ events. The overall mean value of the efficiency at $2200 \mathrm{~V}$ result to be $0.929 \pm 0.008$. This is good enough to reconstruct the projectile track since the BM has 6 planes of cells for each view and, with this efficiency value, the mean number of hits per particle and per view is $5.58 \pm 0.06$, which is high enough compared to the three minimum hits required to reconstruct a track on a view.

In order to evaluate the BM efficiency as a function of the hit drift distance exploiting the external independent detector, the efficiency is measured with a different method using the BM hits and the MSD reconstructed tracks: for each selected event, the MSD track is propagated into the BM detector and the cells crossed by the track are selected and considered as the pivots. Each pivot is associated with a drift distance and a probe counter. The former is evaluated as the distance between the track and the cell sense wire. The latter is equal to one if the cell contains a BM hit and zero if it does not. At the end, all the pivots with the same drift distance are grouped and the relative efficiency is evaluated as the ratio of the number of the associated probes to the number of pivots. The result is shown in the right panel of Fig. 5. The cell efficiency is always larger than $98 \%$ for hit distances below $0.45 \mathrm{~cm}$, where the strength of the electric field ensures good charge collection. At the border of the cell, for distances above $0.6 \mathrm{~cm}$, where the electric field is of $0.55 \mathrm{kV} / \mathrm{cm}$, the efficiency drops below the 0.93 mean value, due to incomplete charge collection. The effect of the detectors misalignment and the track reconstruction precision on the efficiency drop at large drift distance is negligible, since the drift distance of the pivots are evaluated with an accuracy of $0.034 \mathrm{~cm}$ in the worst case scenario of proton beam at $80 \mathrm{MeV}$, where the MCS is maximized. In principle, it would be possible to balance the inefficiency by rising the $\mathrm{HV}$, but the disadvantage is the enhancement of cross talks and delta rays: the number of hits per event would rise above 1213 hits/event and the number of fake hits detected by the BM would increase, as shown in Fig. 3. Moreover, the BM cells are staggered overlapping a cell border with the anode of the cells placed in the adjacent layers, which allows recovering the inefficiency.

\subsection{Spatial and angular resolution}

The BM spatial resolution has been evaluated with two different methods: the first one consists in the extrapolation of the MSD reconstructed tracks into the BM cells and in the evaluation of the residual, by checking the difference between the distance of the track with 

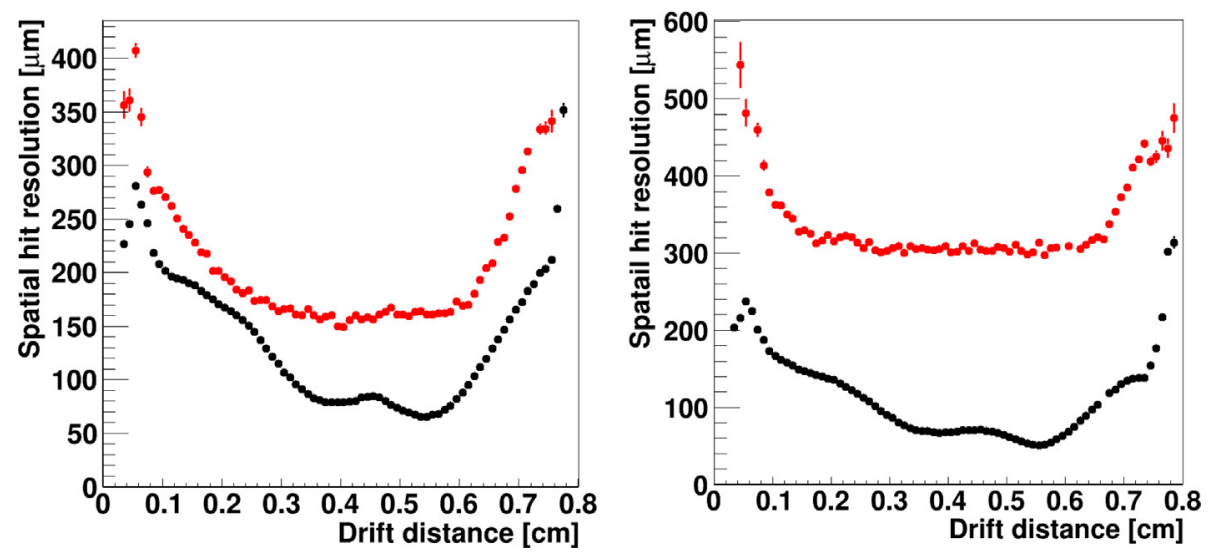

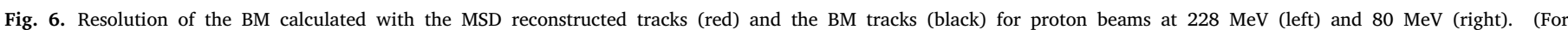
interpretation of the references to color in this figure legend, the reader is referred to the web version of this article.)

the cell center and the BM space measurement. The obtained residual distribution is fitted with a Gaussian and, in order to extrapolate the contribution of the BM detector, the square of the MSD detector resolution of $45 \mu \mathrm{m}$ is subtracted from the fitted variance. The results of this method are shown in red in Fig. 6. For protons at $228 \mathrm{MeV}$ and $80 \mathrm{MeV}$ the mean resolution is $200 \pm 60 \mu \mathrm{m}$ and $340 \pm 60 \mu \mathrm{m}$ while the resolution at the central part of the cell is of the order of 150 and $300 \pm 10 \mu \mathrm{m}$, respectively for the two beam energies. In both cases there is a worsening of the performance at the cell border and close to the cell center. In the former case, it is due to the diffusion process of the ionized particles that leads to a Gaussian smearing of the position of particles with a variance that depends on the drift time. In the latter case, the avalanches produced by the electrons close to the anode wire modify the electric field reducing the resolution at the cell center. Furthermore, for a given fixed time resolution due to the acquisition electronics, the associated space resolution is lower at the cell center since in this region the BM space-time relation is steeper due to the electric field dependence. The second method to evaluate the BM spatial resolution adopts the same strategy as the previous one, but it uses the BM reconstructed tracks instead of the MSD tracks. The resulting mean resolution is $140 \pm 70 \mu \mathrm{m}$ and $120 \pm 60 \mu \mathrm{m}$ for protons at 228 and $80 \mathrm{MeV}$ respectively. In both cases, the resolution in the central part of the cell is in a range between $100 \mu \mathrm{m}$ and $60 \mu \mathrm{m}$ (Fig. 6).

In order to analyze the difference between the results of the two methods, a MC simulation study was performed by means of FLUKA code. The experimental setup shown in Fig. 1 was simulated with a proton beam at $80 \mathrm{MeV}$ and an oxygen beam at $400 \mathrm{MeV} / \mathrm{u}$. The BM spatial resolution was reproduced in the simulations applying a smear on the drift distances according to the resolutions obtained with the BM track method from the data collected with protons at $80 \mathrm{MeV}$. Applying the same procedure as done for the experimental data, the results of the two resolution evaluation methods on the two simulations are shown in Fig. 7. The black and the blue points are the resolutions obtained with the BM track method respectively for the proton and the oxygen ion beams. Both the curves are similar to each other and they are compatible to the input resolution. The results of the MSD track method are indicated by the red and the green points that respectively represent the proton and the oxygen beam simulations. In this case the two curves differ from each other due to the distinct MCS contributions. Protons have lower charge and lower energy with respect to the oxygen ions, so the MCS effect is enhanced and the spatial resolution evaluated with the MSD tracks in the central part of the cell is of the order of 250 $\mu \mathrm{m}$. The difference between the simulated and the experimental data results can be attributed to an underestimate of the material budget in the simulations. Indeed, similar results are obtained also with a simulation performed with protons at $228 \mathrm{MeV}$. In the case of oxygen ions, the MCS effect is limited by the particle higher charge and kinetic

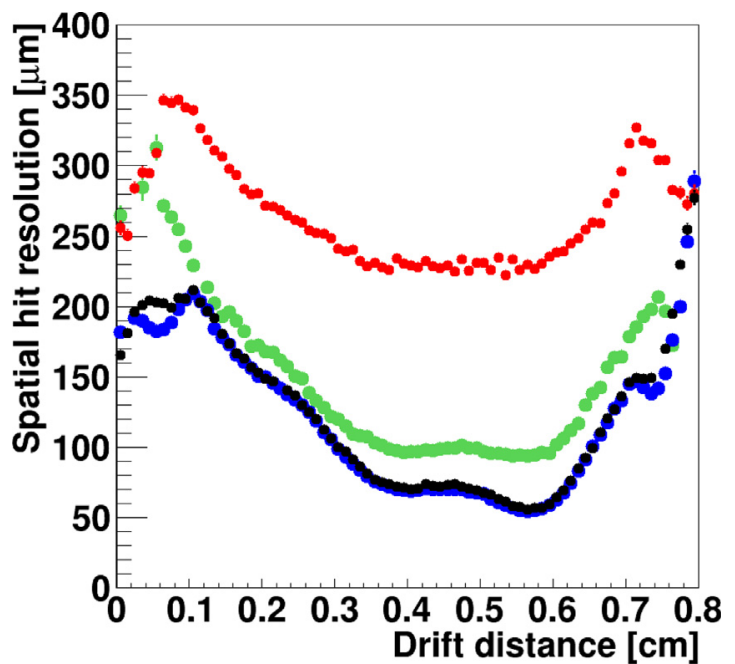

Fig. 7. BM spatial resolutions evaluated by means of MC simulations: the red and the green points represent the resolutions evaluated with the MSD tracks respectively for the simulations performed with protons at $80 \mathrm{MeV}$ and oxygen ions at $400 \mathrm{MeV} / \mathrm{u}$. The resolutions obtained with the BM track method for the two simulations are shown by the black (protons at $80 \mathrm{MeV}$ ) and the blue (oxygen at $400 \mathrm{MeV} / \mathrm{u}$ ) points. (For interpretation of the references to color in this figure legend, the reader is referred to the web version of this article.)

energy. Indeed the spatial resolutions evaluated with the MSD track method are of the order of $100 \mu \mathrm{m}$ in the central part of the cell, that is slightly above the simulation input resolution. Therefore, with an external detector it is possible to evaluate the BM resolution including the worsening due to the MCS given by the BM itself and the air gap between the BM and the two layers of MSD. Alternatively, adopting the BM tracks, it is possible to evaluate the detector intrinsic resolution, that represents the upper limit of the BM performances. This conclusion explains also the better resolutions obtained exploiting the MSD track method with the $228 \mathrm{MeV}$ protons experimental data with respect to the $80 \mathrm{MeV}$ protons data.

In order to evaluate the $\mathrm{BM}$ angular resolution, the distribution of the residuals between the BM and the MSD track slopes has been fitted with a Gaussian function for each dataset and for each view. The resulting $\mathrm{BM}$ angular resolution for the 228 and $80 \mathrm{MeV}$ protons is of $1.62 \pm 0.16 \mathrm{mrad}$ and $2.1 \pm 0.4 \mathrm{mrad}$. On each view the distance along the beam direction between the position of the first and the last layer of sense wires is of $13 \mathrm{~cm}$. Considering this length, the angular resolution is compatible with the previous spatial resolution measurement performed with the MSD tracks. 


\section{Discussion}

The calibration of the space-time relations and the performance assessment of the FOOT BM detector have been carried out with the MSD detectors at the Trento proton therapy facility with proton beams of 228 and $80 \mathrm{MeV}$ kinetic energy. We successfully evaluated the drift chamber space-time relations with an external independent tracking detector. No relevant differences were found between the space-time relations calculated with protons at the two energies. The hit detection efficiency of the BM has been evaluated to be $0.929 \pm 0.008$ on average, with values $>0.98$ for most of the cell region (see Fig. 5). A drop of the efficiency at the cell border has been observed, but a staggering of the cells compensates this effect for tracking purposes.

In the FOOT experiment, the BM will be adopted to reconstruct the projectile direction and the impinging point position on the target material. To this purpose, we investigated the BM angular resolution by evaluating the angular residual distribution between the BM and the MSD tracks. The results show an angular resolution of $1.62 \pm 0.16$ $\mathrm{mrad}$ and $2.1 \pm 0.4 \mathrm{mrad}$ for the higher and lower beam energies, respectively. At the same time, two methods have been developed and studied by means of MC simulations to measure the BM spatial resolution. The first one adopts the information provided by an external tracker (i.e. the MSD detectors) and it takes into account both the BM intrinsic resolution and the MCS effect. The results show a resolution of about 150 and $300 \pm 10 \mu \mathrm{m}$ in the central part of the cell for protons of 228 and $80 \mathrm{MeV}$ of kinetic energy, respectively. The second method exploits instead the BM reconstructed tracks. It depends essentially only on the BM intrinsic resolution and it allows us to determine the detector performance limit, that has been found to be $60-100 \mu \mathrm{m}$ in the central part of the cells. Even if the spatial resolution obtained with the BM tracks are better than the results achieved with the MSD tracks due to the MCS effect, the evaluation of the space-time relations performed with the external detector is still preferred since it avoids the dependence of the results on the BM reconstruction algorithm and the limitations experienced in the past [9] when a self-calibration method was used.

Both the overall hit detection efficiency and the BM intrinsic resolution evaluation method have already been adopted in the FIRST experiment [11]. The results presented in this work are compatible with the previous ones, even if the working point was not the same. Our results show that the detector fulfills the FOOT requirements and it can be adopted for the future FOOT data taking in which the BM will be adopted to reconstruct heavy ion beams with $\mathrm{Z} \geq 2$ with kinetic energies of $200-700 \mathrm{MeV} / \mathrm{u}$.

\section{CRediT authorship contribution statement}

Dong Yunsheng: Formal analysis, Writing - original draft, Investigation, Software, Methodology. Silvestre Gianluigi: Formal analysis, Investigation, Resources. Colombi Sofia: Investigation, Resources, Methodology. Patera Vincenzo: Funding. Battistoni Giuseppe: Supervision, Writing - review \& editing, Investigation, Conceptualization,
Funding, Methodology. Servoli Leonello: Supervision, Writing - review \& editing, Investigation, Conceptualization. Tommasino Francesco: Supervision, Writing - review \& editing, Investigation, Conceptualization, Methodology.

\section{Declaration of competing interest}

The authors declare that they have no known competing financial interests or personal relationships that could have appeared to influence the work reported in this paper.

\section{Acknowledgment}

The FOOT Collaboration is indebted to Mr. Mario Anelli who built the Drift Chamber at the Laboratori Nazionali di Frascati of INFN. All authors reviewed and approved the manuscript.

\section{References}

[1] H. Paganetti, Relative biological effectiveness (RBE) values for proton beam therapy. Variations as a function of biological endpoint, dose, and linear energy transfer, Phys. Med. Biol. 59 (22) (2014) R419, http://dx.doi.org/10.1088/00319155/59/22/R419.

[2] F. Tommasino, M. Durante, Proton radiobiology, Cancers 7 (2015) 353-381, http://dx.doi.org/10.3390/cancers7010353.

[3] J. Dudouet, et al., Double-differential fragmentation cross-section measurements of $95 \mathrm{MeV} /$ nucleon ${ }^{12} \mathrm{C}$ beams on thin targets for hadron therapy, Phys. Rev. C 88 (2013) 024606, http://dx.doi.org/10.1103/PhysRevC.88.024606.

[4] W.R. Webber, J.C. Kish, D.A. Schrier, Individual isotopic fragmentation cross sections of relativistic nuclei in hydrogen, helium, and carbon targets, Phys. Rev. C 41 (1990) 547-565, http://dx.doi.org/10.1103/PhysRevC.41.547.

[5] R. Pleskac, et al., The FIRST experiment at GSI, Nucl. Instrum. Methods Phys. Res. A 678 (2012) 130-138, http://dx.doi.org/10.1016/j.nima.2012.02.020.

[6] J. Alcaraz, et al., The alpha magnetic spectrometer silicon tracker: Performance results with protons and helium nuclei, Nucl. Instrum. Methods Phys. Res. A 593 (3) (2008) 376-398, http://dx.doi.org/10.1016/j.nima.2008.05.015.

[7] F. Tommasino, et al., Proton beam characterization in the experimental room of the Trento Proton Therapy facility, Nucl. Instrum. Methods Phys. Res. A 869 (2017) 15-20, http://dx.doi.org/10.1016/j.nima.2017.06.017.

[8] M. Toppi, et al., Measurement of fragmentation cross sections of ${ }^{12} \mathrm{C}$ ions on a thin gold target with the FIRST apparatus, Phys. Rev. C 93 (06) (2016) 064601, http://dx.doi.org/10.1103/PhysRevC.93.064601.

[9] A. Paoloni, et al., The upstream detectors of the FIRST experiment at GSI, Phys. Proc. 37 (2012) 1466-1472, http://dx.doi.org/10.1016/j.phpro.2012.03.745.

[10] M. Berger, J. Coursey, M. Zucker, J. Chang, Stopping-power and range tables for electrons, protons, and helium ions, http://dx.doi.org/10.18434/T4NC7P.

[11] Z. Abou-Haidar, et al., Performance of upstream interaction region detectors for the FIRST experiment at GSI, J. Instrum. 7 (2012) P02006, http://dx.doi.org/ 10.1088/1748-0221/7/02/P02006.

[12] T. Alexopoulos, et al., Implementation of the Legendre Transform for track segment reconstruction in drift tube chambers, Nucl. Instrum. Methods Phys. Res. A 592 (3) (2008) 456-462, http://dx.doi.org/10.1016/j.nima.2008.04.038.

[13] T. Böhlen, F. Cerutti, M. Chin, A. Fassò, A. Ferrari, P. Ortega, A. Mairani, P. Sala, P. Smirnov, V. Vlachoudis, The FLUKA code: Developments and challenges for high energy and medical applications, Nucl. Data Sheets 120 (2014) 211, http://dx.doi.org/10.1016/j.nds.2014.07.049.

[14] A. Ferrari, P.R. Sala, A. Fassò, J. Ranft, FLUKA: A Multi-Particle Transport Code, Tech. Rep., (CERN-2005-10, INFN/TC_05/11, SLAC-R-773) CERN, INFN, SLAC, 2005, http://dx.doi.org/10.2172/877507.

[15] G. Battistoni, et al., The FLUKA code: An accurate simulation tool for particle therapy, Front. Oncol. 6 (2016) 116, http://dx.doi.org/10.3389/fonc.2016. 00116. 